22. Farooqui, A. A., Taylor, W. A. \& Horrocks, L. A. Characterization and solubilization of membrane bound diacylglycerol lipases from bovine brain. Int. J. Biochem. 18, 991-997 (1986).

23. Jung, M. et al. Characterization of CB1 receptors on rat neuronal cell cultures: binding and functional studies using the selective receptor antagonist SR 141716A. J. Neurochem. 68, 402-409 (1997).

24. Nowicky, A. V. \& Teyler, T. J. The modulation of long-term potentiation by Delta-9-tetrahydrocannabinol in the rat hippocampus, in vitro. Brain Res. Bull. 19, 663-672 (1987).

25. Terranova, J.-P., Michaud, J.-C., Le Fur, G. \& Soubrié, P. Inhibition of long-term potentiation in rat hippocampal slices by anandamide and WIN55212-2: reversal by SR141716 A, selective antagonist of CB1 cannabinoid receptors. Naunyn-Schmiedeberg's Arch. Pharmacol. 352, 576-579 (1995).

26. Heyser, C. J., Hampson, R. E. \& Deadwyler, S. A. Effects of Delta-9-tetrahydrocannabinol on delayed match to sample performance in rats: Alterations in short-term memory associated with changes in task specific firing of hippocampal cells. J. Pharmacol. Exp. Ther. 264, 294-307 (1993).

27. Mallet, P. E. \& Beninger, R. J. The endogenous cannabinoid receptor agonist anandamide impairs memory in rats. Behav. Pharm. 7, 276-284 (1996).

28. Schmid, H. H. O., Schmid, P. C. \& Natarajan, V. The $\mathrm{N}$-acylation-phosphodiesterase pathway and cel signalling. Chem. Phys. Lipids 80, 133-142 (1996).

29. Stella, N., Pellerin, L. \& Magistretti, P. Modulation of the glutamate-evoked release of arachidonic acid from mouse cortical neurons: involvement of a $\mathrm{pH}$-sensitive membrane phospholipase A2. J. Neurosci. 15, 3307-3317 (1995).

30. Schweitzer, P., Madamba, S., Champagnat, J. \& Siggins, G. R. Somatostatin inhibition of hippocampa CAl pyramidal neurons: mediation by arachidonic acid and its metabolites. J. Neurosci. 13, 20332049 (1993).

Acknowledgements. We thank G. R. Siggins for use of equipment (funded by NIMH), S. Madamba for technical help, and P. Magistretti, M. Beltramo and A. Giuffrida for reading the manuscript critically. Thi research was supported by the Neurosciences Research Foundation, which receives major support from Novartis, and by a scientist development award from the National Institute of Drug Abuse (to P.S.).

\section{Placental abnormalities in} mouse embryos lacking the orphan nuclear receptor ERR- $\beta$

\section{Jiangming Luo ${ }^{\star} \dagger$, Robert Sladek ${ }^{\star} \dagger \ddagger$, Jo-Ann Bader ${ }^{\star}$, Annie Matthyssen*, Janet Rossant $\ddagger \S$ \& Vincent Giguère $* \|$}

${ }^{*}$ Molecular Oncology Group, Royal Victoria Hospital, 687 Pine Avenue West, Montreal, Québec H3A 1A1, Canada

$\ddagger$ Department of Molecular and Medical Genetics, University of Toronto, Toronto, Ontario M5S 1A8, Canada

$\$$ Samuel Lunenfeld Research Institute, Mount Sinai Hospital, 600 University Avenue, Toronto, Ontario M5G 1A8, Canada

$\|$ Departments of Biochemistry, Oncology and Medicine, McGill University, Montreal, Québec H3G 1Y6, Canada

$\dagger$ These authors contributed equally to this work.

Classical endocrine studies have shown that steroid hormones are required for the maintenance of pregnancy and placental viability. The oestrogen-receptor-related receptor $\beta$ (ERR- $\beta$ ) is an orphan member of the superfamily of nuclear hormone receptors ${ }^{1}$. Although ERR- $\beta$ is homologous to the oestrogen receptor and binds the oestrogen response element ${ }^{2}$, it is not activated by oestrogens ${ }^{1}$. Expression of ERR- $\beta$ during embryogenesis defines a subset of extra-embryonic ectoderm that subsequently forms the dome of the chorion, suggesting that ERR- $\beta$ may be involved in early placental development. Homozygous mutant embryos generated by targeted disruption of the Estrrb gene have severely impaired placental formation, and die at $\mathbf{1 0 . 5}$ days post-coitum. The mutants display abnormal chorion development associated with an overabundance of trophoblast giant cells and a severe deficiency of diploid trophoblast. The phenotype can be rescued by aggregation of Estrrb mutant embryos with tetraploid wildtype cells, which contribute exclusively to extra-embryonic tissues. Our results indicate that ERR- $\beta$ has an important role in early placentation, and suggest that an inductive signal originating from or modified by the chorion is required for normal trophoblast proliferation and differentiation.

During early embryonic development in the mouse, expression of the gene encoding ERR- $\beta$ is restricted to extra-embryonic tissues (Fig. 1). ERR- $\beta$ transcripts are first detected in a subset of cells in extra-embryonic ectoderm at 5.5 days post-coitum (d.p.c.) and become more prominently expressed at 6.0 d.p.c. (Fig. 1a-d). At 6.5 d.p.c., ERR- $\beta$ is expressed by ectodermally derived regions of the amniotic fold (Fig. 1e, f); these cells then form the chorion, where ERR- $\beta$ expression is present at 7.5 d.p.c. (Fig. 1g, h). ERR- $\beta$ expression diminishes as the chorion fuses with the ectoplacental cone, and by 8.5 d.p.c. ERR- $\beta$ transcripts are present only at the free margin of the chorion (Fig. 1i, j). ERR- $\beta$ transcripts are not detected in extra-embryonic tissue at subsequent stages of development. This highly specific expression pattern suggests that ERR- $\beta$ may be important for the formation or function of the chorion.

To assess the role of ERR- $\beta$ in placental development, we have created homozygous null Estrrb mutants by homologous recombination. Targeted disruption of the Estrrb gene was achieved in R1 embryonic stem (ES) cells by replacement of the second Estrrb exon with a neomycin phosphotransferase cassette (Fig. 2a). Two independent clones containing the predicted mutant allele produced male chimaeric mice that transmitted the mutation through the germ line (Fig. 2b). Mice heterozygous for the mutation were viable and were bred to generate homozygous mutants. No homozygotes were found in 62 live-born progeny of heterozygous intercrosses, indicating that the Estrrb mutation caused embryonic lethality. Embryos were genotyped at various stages of gestation to determine when fetal death occurred; viable homozygous mutants were not detected later than 10.5 d.p.c. (Fig. 2c and Table 1). Embryos examined at 9.5 d.p.c. demonstrated growth failure as well as absence of chorioallantoic fusion (Fig. 3a). At age 10.5 d.p.c., $E_{s t r r}{ }^{-l-}$ embryos had no detectable heartbeat and had started to undergo resorption (Fig. 3b). These results indicate that the Estrrb mutation was lethal at 9.5-10.5 d.p.c., and that Estrrb ablation was associated with abnormal placental development.

Histological examination showed that embryonic and extraembryonic tissues in Estrrb homozygous mutants were normal at 6.5 d.p.c. (Fig. 3c, d). In particular, development of the ectoplacental cone and the extra-embryonic ectoderm appeared normal in the mutant embryos. At 7.5 d.p.c., the chorion of the Estrrb homozygous mutants was either absent or hypoplastic and the embryos displayed varying amounts of growth retardation (Fig. 3d, f). In all cases, the extra-embryonic tissues appeared viable. At 8.5 d.p.c., abnormalities were seen in the development of early placenta and the ectoplacental cone, as the Estrrb mutants showed a marked increase in the number of secondary giant cells accompanied by a near absence of diploid trophoblast cells. Further, chorioallantoic fusion did not occur, and the placenta was absent or markedly decreased in size (Fig. $3 \mathrm{~g}-\mathrm{j}$ ). Although most of the mutant embryos at 8.5 d.p.c. did not show obvious abnormalities, the mutants with the most marked placental abnormalities tended to be smaller and to have disorganized internal structure. By 9.5 d.p.c., the mutant placentas contained multiple layers of giant cells with no labyrinthine trophoblast or spongiotrophoblast (Fig. 3k-n). Mice derived from two independent targeted ES cell lines studied in C57BL, CD1 and 129/Sv genetic backgrounds displayed identical phenotypes (data not shown).

To confirm the histological findings in Estrrb mutant trophoblast tissues, expression of several markers of trophoblast differentiation

\begin{tabular}{|c|c|c|c|c|}
\hline Stage & Total & $+/+$ & $\begin{array}{c}\text { Genotype } \\
+/-\end{array}$ & $-1-$ \\
\hline E7.5 & 81 & 20 & 41 & 20 \\
\hline E8.5 & 134 & 33 & 64 & 37 \\
\hline E9.5 & 40 & 7 & 24 & $9 *$ \\
\hline E10.5 & 44 & 14 & 22 & $8^{*}$ \\
\hline E11.5 & 46 & 14 & 32 & 0 \\
\hline E12.5 & 10 & 3 & 7 & 0 \\
\hline Term & 8 & 4 & 4 & 0 \\
\hline P28 & 54 & 19 & 35 & 0 \\
\hline
\end{tabular}

E, embryonic day number; $\mathrm{P}$, postnatal day number *Abnormal embryos. 


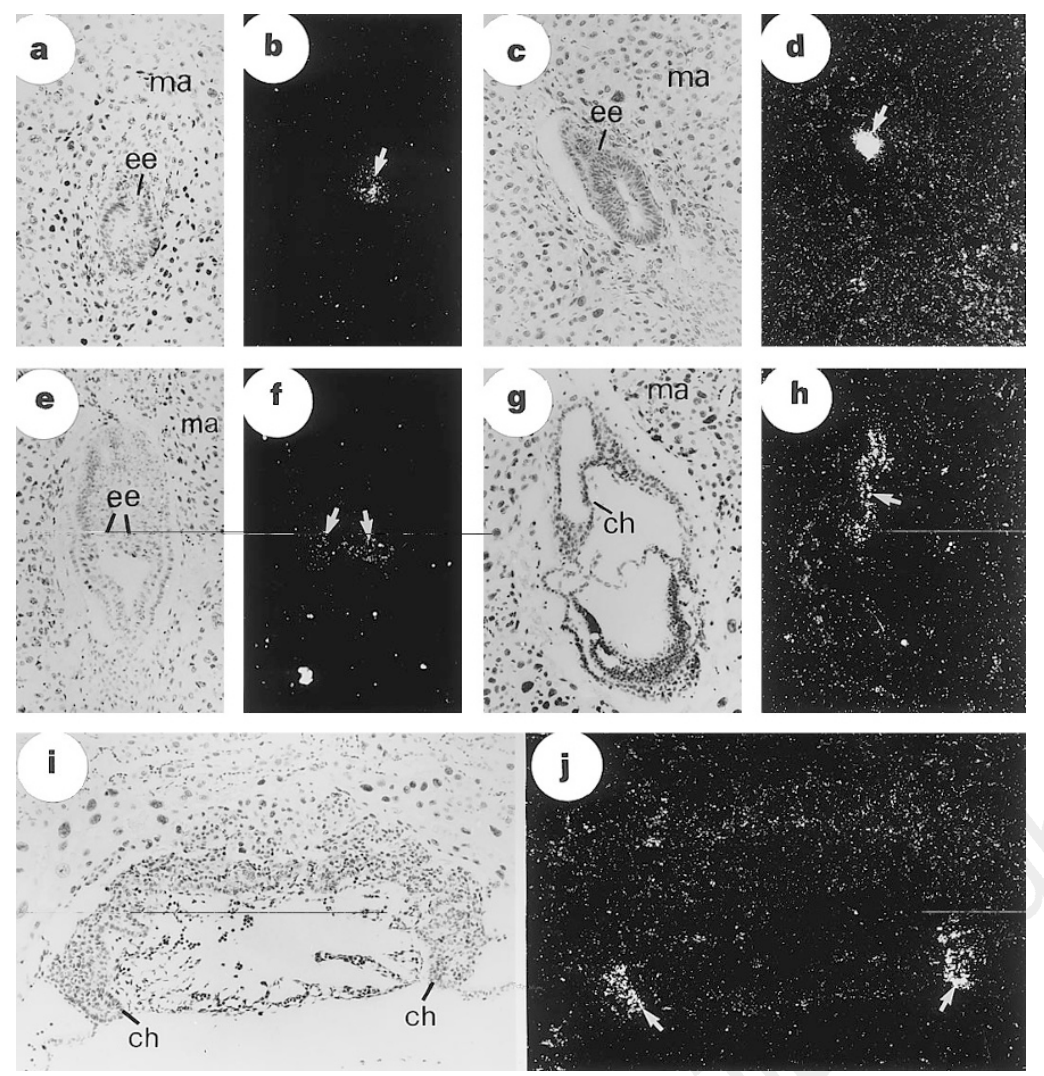

Figure 1 Expression of Estrrb in trophoblast tissues. Bright-field images of normal embryos at $5.5(\mathbf{a}), 6.0$ (c), $6.5(\mathbf{e}), 7.5(\mathbf{g})$ and 8.5 (i) d.p.c. RNA in situ hybridization was performed using a 320-bp riboprobe specific for Estrrb exon 2. Estrrb transcripts are present in the extra-embryonic ectoderm at 5.5 (b), 6.0 (d) and 6.5 (f) d.p.c. At 7.5 d.p.c., Estrrb transcripts are exclusively detected in chorion (h), and at 8.5 d.p.c. are present only at its free margin (j). Estrrb expression was not detected in the ectoplacental cone at any stage of development, nor was placental expression detected after 8.5 d.p.c. Arrows indicate regions of specific hybridization; ch, chorion; ee, extra-embryonic ectoderm; ma, maternal decidua.

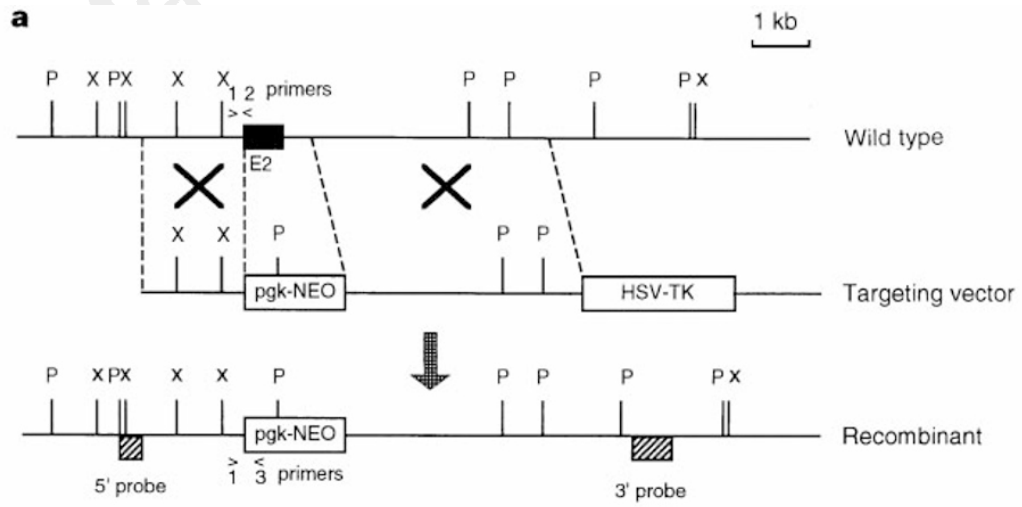

b

c

d

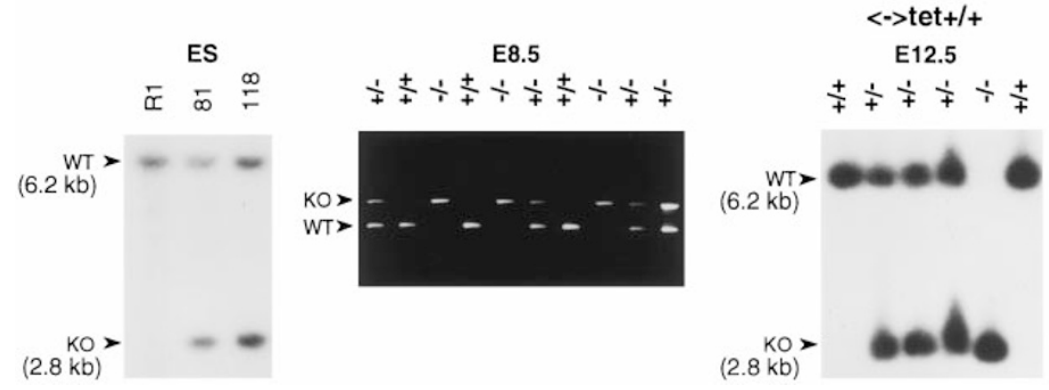

Figure 2 Targeted disruption of the Estrrb gene and heterozygote inbreeding analysis. a, Schematic representation of the ERR- $\beta$ locus (wild type, top), targeting vector (middle), and targeted allele (recombinant, bottom). Arrows indicate the positions of primers P1, P2 and P3 used to genotype the offspring of heterozygous matings. Shaded rectangles represent the $5^{\prime}$ and $3^{\prime}$ probes used for Southern analysis of ES cell colonies. P, Pstl; X, Xhol. b, Southern blot analysis of targeted ES clones. DNA from parental ES cells (R1) and targeted clones (81 and 118) was digested with Pstl and hybridized to the 5 ' probe. The positions of bands corresponding to the wild-type allele (WT, $6.2 \mathrm{~kb}$ ) and the mutant allele (KO, $2.8 \mathrm{~kb}$ ) are indicated. c, PCR analysis of genotypes of embryos at 8.5 d.p.c. from a heterozygote intercross. d, ERR- $\beta$ mutant embryos are present among the progeny at 12.5 d.p.c. of heterozygous intercross rescued by tetraploid aggregation. 
was examined by RNA in situ hybridization. Expression of the Mash-2 gene, which is a marker of spongiotrophoblast, labyrinthine trophoblast cells and their precursors ${ }^{3}$, was examined in Estrrb mutant embryos. At 7.5 d.p.c., Mash-2 expression was not changed in the trophoblast region of Estrrb ${ }^{-1}$ embryos (data not shown). Diploid trophoblast cells expressing the Mash-2 gene were reduced in number at 8.5 d.p.c. (Fig. $4 \mathrm{a}-\mathrm{d}$ ) and absent at 9.5 d.p.c. in the $E_{\text {strrb }}{ }^{-1-}$ trophoblasts (Fig. $4 \mathrm{~g}-\mathrm{j}$ ). Similar results were obtained with the markers 4311 (ref. 4) and Flt-1 (ref. 5), which have expression restricted to the spongiotrophoblast and its precursors (data not shown). In contrast, expression of transcripts for the trophoblast giant-cell markers placental lactogen-1 (ref. 6) and proliferin (ref. 7; data not shown) were markedly increased at both 8.5 d.p.c. (Fig. $4 \mathrm{e}, \mathrm{f})$ and 9.5 d.p.c. (Fig. $4 \mathrm{k}, \mathrm{l}$ ). To determine when diploid
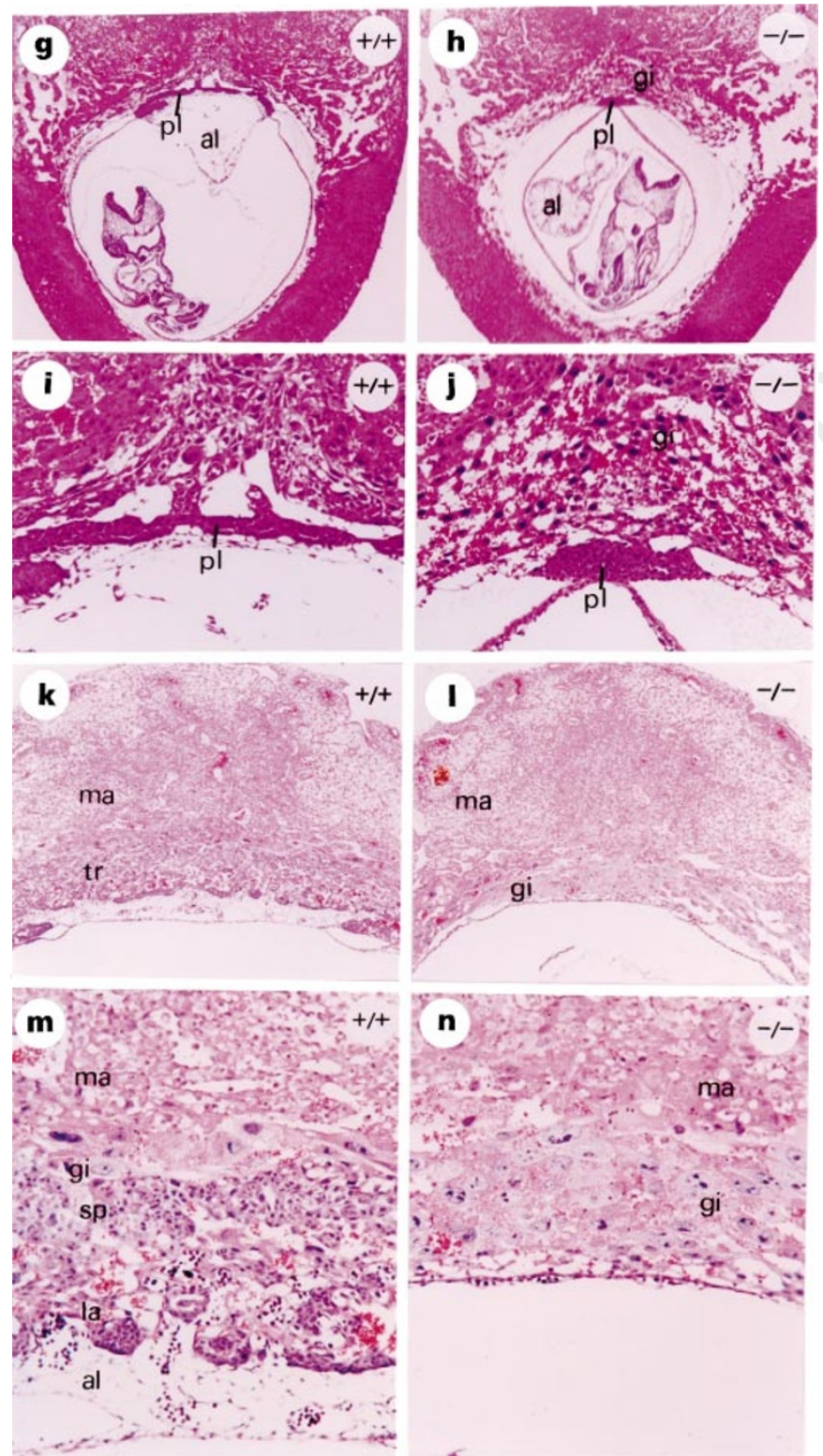

trophoblast proliferation in Estrrb mutants became impaired, we examined the incorporation of 5-bromo-2'-deoxyuridine (BrdU) into DNA in trophoblast cells at different stages of development. At 7.5 d.p.c., the fraction of diploid trophoblast cells incorporating BrdU was similar in wild-type and mutant ectoplacental cones (approximately $85 \%$ of cells). However, at 8.0 and 8.5 d.p.c. The fraction of Estrrb ${ }^{-/-}$diploid trophoblast cells incorporating BrdU was less than half that of normal trophoblast (between $30 \%$ and $40 \%$ of cells). Taken together, these results confirmed the histological observations and demonstrated that reduced proliferation of diploid trophoblast cells first occurred a few hours after failure of chorion development.

By 9.5 d.p.c., Estrrb ${ }^{-1-}$ embryos displaced impaired development, as would be expected if placental function were significantly
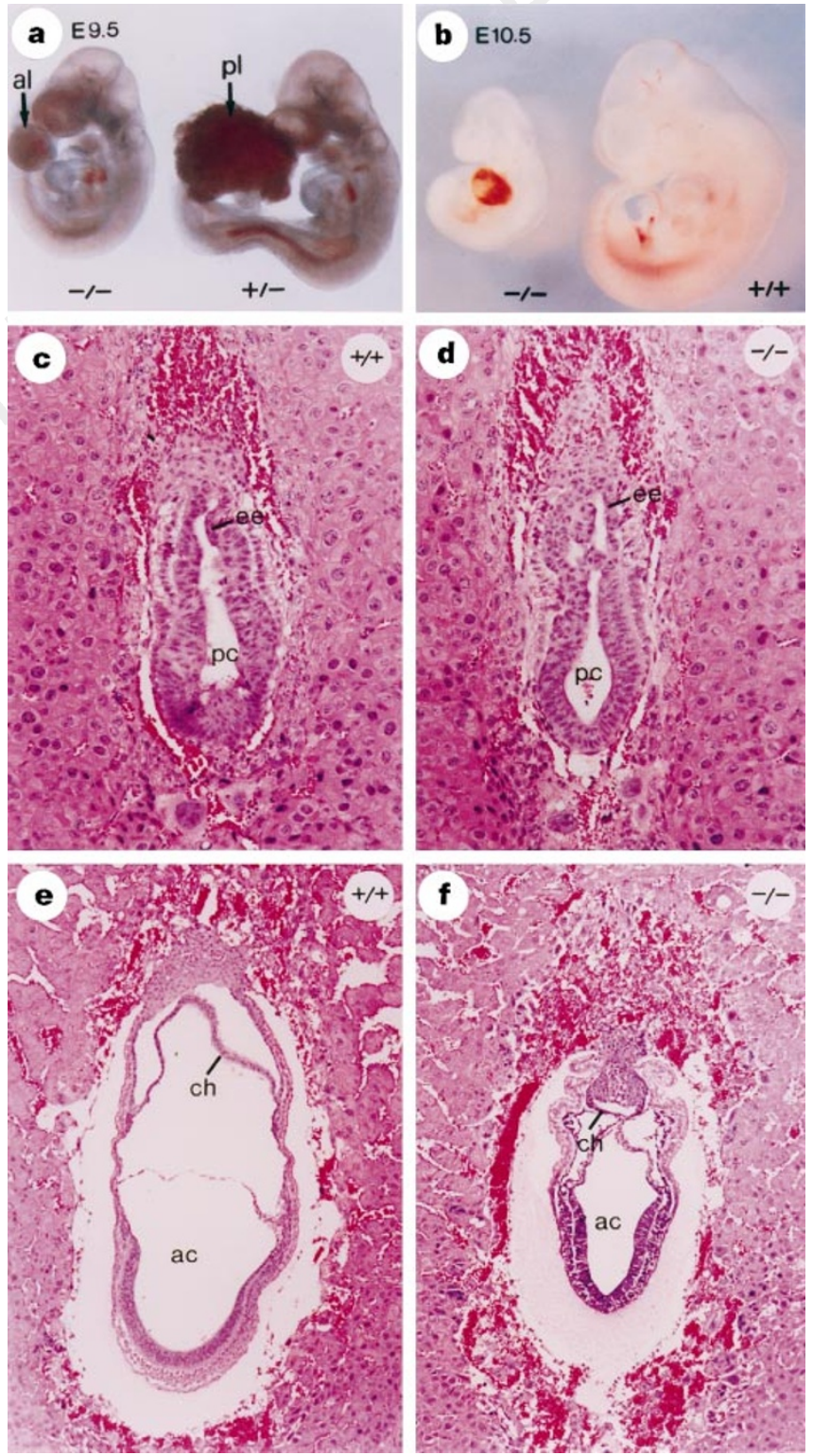

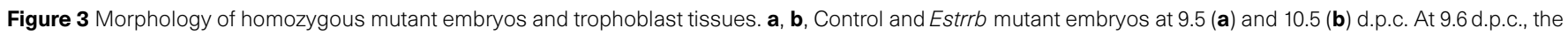

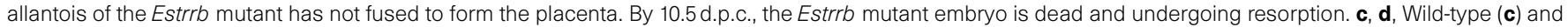

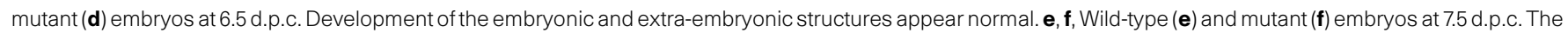

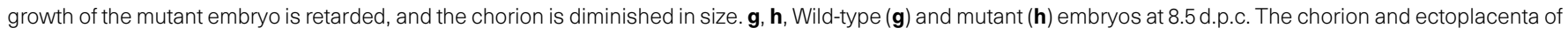

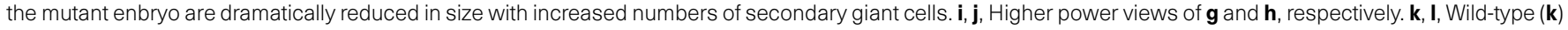

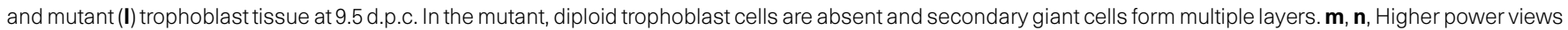

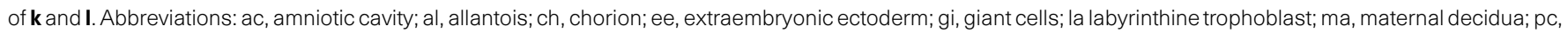
proamniotic cavity; pl, placenta; sp, spongiotrophoblast; tr, trophoblast. 
impaired. Expression of ERR- $\beta$ during early fetal development was present in specific regions of extra-embryonic ectoderm, although no ERR- $\beta$ transcripts were detected in the embryo. This suggested that embryonic growth failure resulted from changes in placental function, a hypothesis that could be tested by performing tetraploid embryo rescue experiments ${ }^{8,9}$. Diploid morulae from Estrrb ${ }^{+/}$ intercrosses were aggregated with four-cell-stage wild-type tetraploid embryos, and the resulting chimaeras were transferred to CD1 foster mothers. In these experiments, wild-type tetraploid cells cannot contribute to embryonic cell lineages, but can contribute to trophoblast lineages, where they might compensate for defects present in the Estrrb ${ }^{-l-}$ offspring. Because Estrrb ${ }^{-/-}$embryos die by 10.5 d.p.c., the pregnancies were allowed to continue to 12.5 d.p.c., when the embryos were genotyped (Fig. 2d). The offspring of four independent rescue experiments included eight Estrrb ${ }^{-/-}$embryos (19\% of the recovered embryos). In one experiment, the tetraploid component was marked by ubiquitous expression of the Escherichia coli $\beta$-galactosidase gene. This provided visual confirmation that all of the trophoblast cells were wild type, and therefore blue, in rescued -/- embryos. The Estrrb ${ }^{-1}$ embryos were externally indistinguishable from their Estrrb ${ }^{+/+}$littermates. These experiments indicate that the early embryonic growth retardation and lethality results solely from abnormal placental development in the Estrrb ${ }^{-1-}$ embryos.

We have shown that the nuclear hormone receptor ERR- $\beta$ is essential for normal placental formation. Ablation of ERR- $\beta$ in extra-embryonic ectoderm results in abnormal chorion formation followed by failure of diploid trophoblast self-renewal, increased formation of giant cells, placental failure and embryonic death. Impaired diploid trophoblast proliferation has been observed when ectoplacental cone fragments are cultured in vitro ${ }^{10}$ or grown ectopically ${ }^{11}$. Trophoblast proliferation could be at least partly restored by transplanting ectoplacental cone fragments into the amniotic cavities of embryos at 7.5 d.p.c.; however, these growth conditions incompletely suppressed the formation of giant cells, suggesting that additional signals from extra-embryonic tissues were required for normal trophoblast development ${ }^{12,13}$. ERR- $\beta$ is expressed in only a subset of diploid trophoblast cells making up the chorion, and disruption of its function causes widespread failure of diploid trophoblast proliferation, which occurs only after impaired formation of chorion. It is therefore possible that ERR- $\beta$ mediates some critical function of the chorion in producing or modulating a diffusible signal that is necessary to support proliferation and block terminal differentiation of the diploid trophoblast. Common methods of influencing fertility have used drugs that target the oestrogen and progesterone receptors ${ }^{14,15}$. As the classic targets of these hormonal agents do not seem to play a unique role in regulating implantation and placentation ${ }^{16,17}$, identification of more specific means of influencing fertility could significantly increase the reproductive health of women. Pharmacological modulation of the activity of ERR- $\beta$, a nuclear hormone receptor that plays an essential role in early placentation, may provide means by which this may be accomplished.

\section{Methods}

Targeted disruption of the Estrrb locus. The targeting vector was constructed using genomic DNA from a $129 / \mathrm{Sv}$ mouse strain. The 1.8-kilobase upstream region and the $4.1-\mathrm{kb}$ downstream region of exon 2 were included in the targeting vector. The targeting vector replaces the first zinc-binding motif of the DNA-binding domain of the ERR- $\beta$ protein with a neo cassette. The linearized construct was electroporated into R1 ES cells ${ }^{18}$, which were then selected with G418 $\left(150 \mu \mathrm{g} \mathrm{ml}^{-1}\right)$ and gancyclovir $(2 \mu \mathrm{M})$. Selected ES colonies were screened by probing PstI-digested ES cell DNA with the $5^{\prime}$ external probe, which hybridized to a $6.2-\mathrm{kb}$ restriction fragment in the wild-type allele and 2.8-kb fragment in the knockout allele. We obtained 12 correctly targeted clones from 165 double-resistant colonies. Single integration of the targeting construct in these ES cell clones was confirmed by reprobing the Southern blots with a neo probe (data not shown). Appropriate integration of the $3^{\prime}$ end of the targeting construct was verified using Southern blot analysis of XhoI-digested DNA using the $3^{\prime}$ external probe (data not shown). Three clones were injected into CD1 blastocysts to generate chimaeras. Chimaeric males from two independent clones (81 and 118) passed the mutation to their offspring. PCR analysis of genotypes of embryos from a heterozygote intercross was performed using primers located in Estrrb intron 1 (P1, 5' TCTTGCCATCCTTAGGCCAGC-3'), the 5' region of Estrrb exon 2 (P2, $5^{\prime}$-AACTAGAGCCCAGGTGCCTGT-3') and the neo cassette (P3, 5' CCTCTGAGCCCAGAAAGCGAA- $3^{\prime}$ ). DNA was obtained from embryonic tissue scraped from histological sections or from the yolk sac. Primers P1
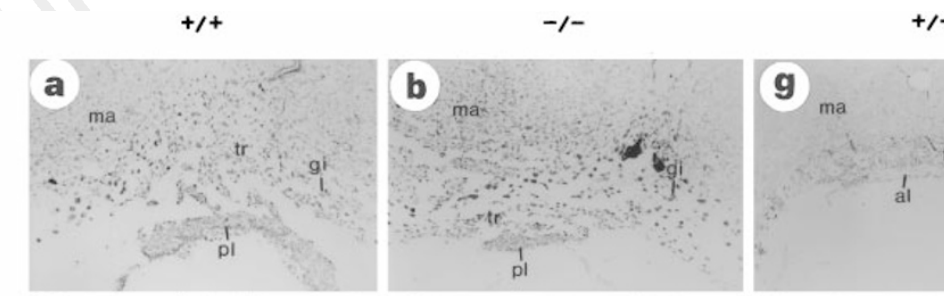

$+1+$

g
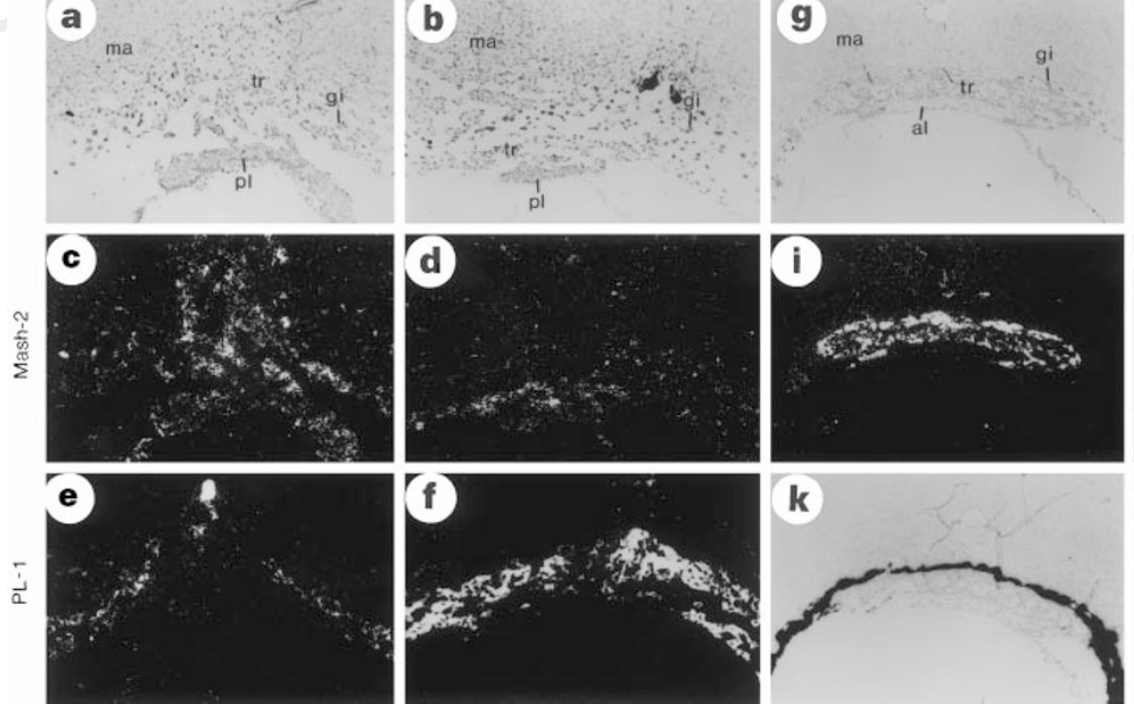
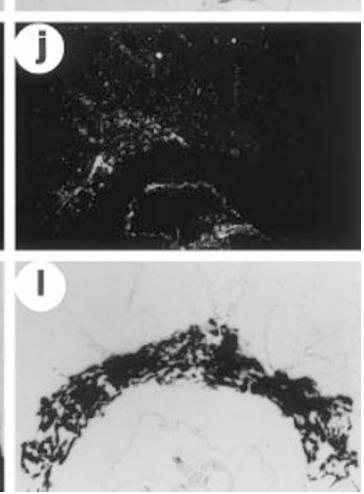

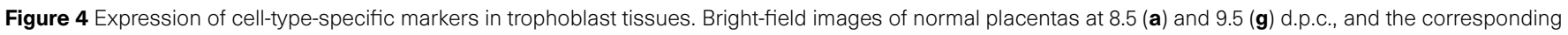

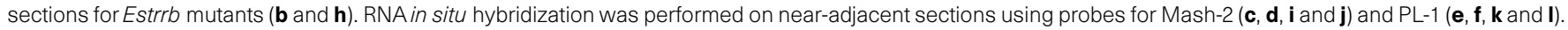

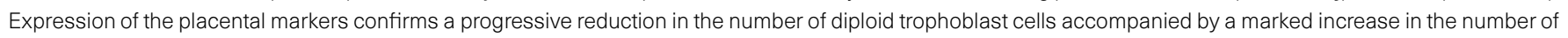
trophoblast giant cells between 8.5 and 9.5 d.p.c.. Abbreviations: al, allantois; gi, giant cells; ma, maternal decidua; pl, placenta; tr, trophoblast. 
and P2 amplify a fragment of 291 base pairs from the wild-type allele; primers P1 and $\mathrm{P} 3$ amplify a product of $440 \mathrm{bp}$, indicating the presence of the targeted allele. Histological and in situ hybridization studies. Embryos and trophoblast tissues from heterozygous intercrosses were fixed in $4 \%$ paraformaldehyde, dehydrated and embedded in paraffin. Sections $(7 \mu \mathrm{m})$ were stained with haematoxylin and $\operatorname{eosin}^{19}$ or processed for in situ hybridization with radiolabelled riboprobes ${ }^{20}$. Genotypes of embryos were identified by PCR using DNA from embryonic tissues scraped from histological sections.

BrdU labelling of trophoblast tissues. BrdU $(100 \mu \mathrm{g}$ per gram of body weight) was injected intraperitoneally into pregnant female mice. The mice were killed $1 \mathrm{~h}$ later, and the embryos and trophoblast tissues were removed and fixed in $4 \%$ paraformaldehyde and processed for immunohistochemistry. The genotype of each embryo was identified by PCR using DNA from embryonic tissues scraped from sections. Sections were incubated with an anti-BrdU monoclonal antibody ${ }^{21}$ (BROMO-2), and antibody binding was visualized with anti-mouse immunoglobulin-alkaline phosphatase (Boehringer Mannheim). Sections were stained with haematoxylin to visualize nuclei and allow cells to be counted.

Generation of tetraploid aggregation chimaeras. Electrofusion of two-cellstage blastomeres collected from CD1 female mice or CD1 females crossed with male homozygous for the ROSA-26 gene trap insertion ${ }^{22}$ was used to produce wild-type tetraploid embryos. The fused embryos were cultured overnight in embryo culture medium in $5 \% \mathrm{CO}_{2}$ at $37^{\circ} \mathrm{C}$. Eight-cell-stage diploid embryos were recovered from the oviduct of embryonic-day (e) 2.5 pregnant ERR- $\beta$ heterozygote females mated to an ERR- $\beta$ heterozygote male. Two four-cell tetraploid embryos were sandwiched around a diploid eight-cell-stage embryo from the heterozygous cross and aggregated overnight. Successfully aggregated embryos were transferred to e2.5-pseudopregnant CD1 recipients. Recipients were killed at e12.5 and embryos were genotyped and examined. Those aggregates made with ROSA-26 tetraploid embryos were also stained for $\beta$-galactosidase activity in the placenta, yolk sac and embryonic components $^{23}$.

Received 21 March; accepted 15 May 1997.

1. Giguère, V., Yang, N., Segui, P. \& Evans, R. M. Identification of a new class of steroid hormon receptors. Nature 331, 91-94 (1988).

2. Pettersson, K. et al. Expression of a novel member of estrogen response element-binding nuclea receptors is restricted to the early stages of chorion formation during mouse embryogenesis. Mech. Dev. 54, 211-223 (1996).

3. Guillemot, F., Nagy, A., Auerbach, A., Rossant, J. \& Joyner, A. L. Essential role of Mash-2 in extraembryonic development. Nature 371, 333-336 (1994)

4. Lescesin, K. R., Vamuza, S. \& Rossant, J. Isolation and characterization of a novel trophoblast-specific cDNA in the mouse. Genes Dev. 2, 1639-1646 (1988).

Finnerty, H. et al. Molecular cloning of murine FLT and FLT4. Oncogene 8, 2293-2298 (1996).

6. Colosi, P., Swiergiel, J. J., Wilder, E. L., Oviedo, A. \& Linzer, D. I. Characterization of proliferin-related protein. Mol. Endocrinol. 2, 579-586 (1988)

Linzer, D. I., Lee, S. J., Ogren, L., Talamantes, F. \& Nathans, D. Identification of proliferin mRNA and protein in mouse placenta. Proc. Natl Acad. Sci. USA 82, 4356-4359 (1985).

8. Nagy, A., Rosant, J., Nagy, R., Abramow-Newerly, W. \& Roder, J. C. Derivation of completely cell culture-derived mice from early-passage embryonic stem cells. Proc. Natl Acad. Sci. USA 90. 8424 8428 (1993).

. Nagy, A. et al. Embryonic stem cells alone are able to support fetal development in the mouse. Development 110. 815-821 (1990).

10. Ilgren, E. B. On the control of the trophoblastic giant-cell transformation in the mouse: homotypic cellular interactions and polyploidy. J. Embryol. Exp. Morphol. 62, 183-202 (1981).

11. Rossant, J. \& Ofer, L. Properties of extra-embryonic ectoderm isolated from postimplantation mouse embryos. J. Embryol. Exp. Morphol. 39, 183-194 (1977).

12. Rossant, J. \& Tamura-Lis, W. Effect of culture conditions on diploid to giant-cell transformation in postimplantation mouse trophoblast. J. Embryol. Exp. Morphol. 62, 217-227 (1981).

13. Ilgren, E. B. Control of trophoblast growth. Placenta 4, 307-328 (1983)

14. Yen, S. S. C. \& Jaffe, R. B. Reproductive Endocrinology (Saunders, Philadelphia, 1991).

15. Speroff, L., Glass, R. H. \& Kase, N. G. Clinical Gynecologic Endocrinology and Infertility (Williams \& Wilkins, Baltimore, 1994)

16. Lubahn, D. B. et al. Alteration of reproductive function but not prenatal sexual development after disruption of the mouse estrogen receptor. Proc. Natl Acad. Sci. USA 90, 11162-11166 (1993).

17. Lydon, J. P. et al. Mice lacking progesterone receptor exhibit pleiotropic reproductive abnormalities. Genes Dev. 9, 2266-2278 (1995)

18. Nagy, A. \& Rossant, J. in Gene targeting: a practical approach (ed. Joyner, A. L.) 147-169 (Oxford Univ Press, 1993).

19. Kaufman, M. H. The Atlas of Mouse Development (Academic, London, 1992).

20. Wilkinson, D. G. In situ Hybridization: a Practical Approach (IRL, Oxford, 1992).

21. Paramithiotis, E. \& Ratcliffe, M. J. B cell emigration directly from the cortex of lymphoid follicles in the bursa of Fabricus. Eur. J. Immunol. 24, 458-463 (1994).

22. Friedrich, G. \& Soriano, P. Promoter traps in embryonic stem cells: a genetic screen to identify and mutate developmental genes in mice. Genes Dev. 5, 1513-1523 (1991).

23. Gossler, A., Joyner, A. L., Rossant, J. \& Skarnes, W. C. Mouse embryonic stem cells and reporte constructs to detect developmentally regulated genes. Science 244, 463-465 (1989).

Acknowledgements. We thank M. Tremblay for injection of ES cells; M. Ratcliffe for the anti-BrdU antibody; and K. Kemball for technical advice. J.L. was supported by a fellowship from the MRC of
Canada, and R.S. was supported by an NCIC Terry Fox clinician-scientist fellowship. This work was supported by grants from the MRC of Canada (C.G., J.R.). J.R. is an international scholar of the Howard Hughes Medical Institute and a Terry Fox research scientist of the NCIC. V.G. is an MRC scientist.

Correspondence and requests for materials should be addressed to V.G. (e-mail: vgiguere@dir.molonc. mcgill.ca).

\section{Inflammatory stimuli induce accumulation of MHC class II complexes on dendritic cells}

\author{
Marina Cella, Anneke Engering, Valerie Pinet* \\ Jean Pieters \& Antonio Lanzavecchia \\ Basel Institute for Immunology, Grenzacherstrasse 487, CH 4005 Basel, \\ Switzerland \\ * Laboratoire d'Immunologie, Inserm U-291, Hôpital Saint Eloi, 34295 \\ Montpellier, France
}

Dendritic cells have the remarkable property of presenting any incoming antigen ${ }^{1}$. To do so they must not only capture antigens with high efficiency and broad specificity, but must also maximize their capacity to load class II molecules of the major histocompatibility complex (MHC) with antigenic peptides in order to present a large array of epitopes from different proteins, each at a sufficient copy number. Here we show that formation of peptide-MHC class II complexes is boosted by inflammatory stimuli that induce maturation of dendritic cells. In immature dendritic cells, class II molecules are rapidly internalized and recycled, turning over with a half-life of about 10 hours. Inflammatory stimuli induce a rapid and transient boost of class II synthesis, while the half-life of class II molecules increases to over 100 hours. These coordinated changes result in the rapid accumulation of a large number of long-lived peptide-loaded MHC class II molecules capable of stimulating $T$ cells even after several days. The capacity of dendritic cells to load many antigenic peptides over a short period of initial exposure to inflammatory stimuli could favour presentation of infectious antigens.

A key feature of dendritic cells is that they can capture antigens in peripheral tissues and migrate to secondary lymphoid organs where they acquire the ability to stimulate naive $\mathrm{T}$ cells ${ }^{1}$. This maturation process was originally noted in freshly isolated Langerhans cells which, upon culture in vitro, lost acidic organelles and the capacity to synthesize class II molecules and to present soluble antigen, while acquiring the ability to stimulate $\mathrm{T}$ cells ${ }^{1-6}$. Although the final results of the maturation process is loss of antigen-capturing and -processing capacity, it is not known whether the generation of peptideMHC class II complexes is enhanced during the transition from immature to mature dendritic cells.

We previously described an in vitro culture system suitable for analysing the maturation process of dendritic cells. Human monocytes cultured in medium supplemented with granulocyte-macrophage colony-stimulating factor (GM-CSF) and interleukin (IL)-4 develop into a homogeneous population with the characteristics of immature dendritic cells, namely high endocytic activity and a low capacity for stimulating $\mathrm{T}$ cells. When challenged by inflammatory stimuli such as tumour-necrosis factor (TNF)- $\alpha$, IL-1 and lipopolysaccharide (LPS), these cells can no longer endocytose, they upregulate adhesion and co-stimulatory molecules, and become mature stimulatory dendritic cells ${ }^{7,8}$. We used this system to study the effect of dendritic cell maturation on class II-restricted antigen presentation.

Cell-surface and total class II molecules were quantified in intact and permeabilized cells. As shown in Fig. 1a, immature dendritic cells express high levels of total class II molecules, but only a small fraction is present on the cell surface. During the first 24 hours after 